PSS $\quad \begin{aligned} & \text { PROCEEDINGS } \\ & \text { OF SCIENCE }\end{aligned}$

\title{
Janusz Kałużny (1955-2015): career and activities
}

\section{Marek J. Sarna ${ }^{1}$}

Nicolaus Copernicus Astronomical Center

Polish Academy of Sciences

Bartycka Str. 18, 00-716 Warszawa, Poland

E-mail: sarna@camk.edu.pl

\section{Michał Różyczka}

Nicolaus Copernicus Astronomical Center

Polish Academy of Sciences

Bartycka Str. 18, 00-716 Warszawa, Poland

E-mail: mnr@camk.edu.pl

We pay homage to the memory of Janusz Kałużny, accomplished photometrist, member of the Salt Science Working Group, founder and leader of the CASE project, a person truly warm and friendly.

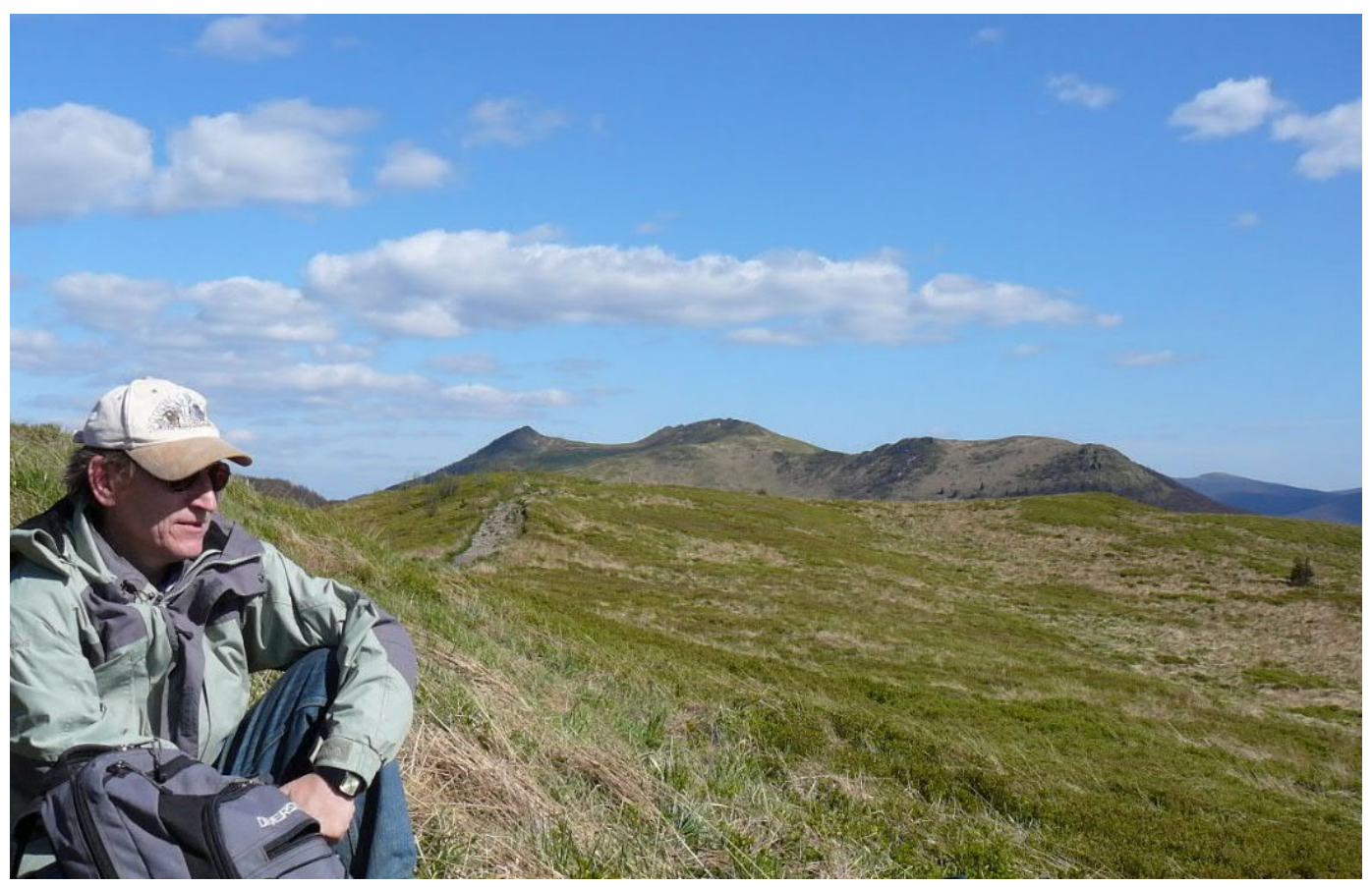

SALT Science Conference 2015 -SSC2015-

1-5 June, 2015

Stellenbosch Institute of Advanced Study, South Africa

${ }^{1}$ Speaker

(c) Copyright owned by the author(s) under the terms of the Creative Commons

Attribution-NonCommercial-NoDerivatives 4.0 International License (CC BY-NC-ND 4.0). 


\section{Education and career}

Janusz was born in Warsaw, but he spent the first years of his life in Świnoujście, a small town in north-western Poland at the Baltic sea. He developed interest in astronomy while still in high school, from which he graduated in 1974. In the same year he began studying astronomy at the University of Warsaw. He obtained his M. Sc. in 1980 - the same year, in which the Solidarity Movement swept through Poland. The country was in its deepest post-war economical crisis, and doing astronomy sounded totally abstract. In fact, a few brightest Polish astronomers emigrated to the West. Those who stayed were soon to face the martial law introduced at the end of 1981. A special permit had to be obtained each time one of the astronomers intended to visit the Ostrowik observing station some $30 \mathrm{~km}$ from Warsaw, where a $60 \mathrm{~cm}$ telescope was housed. Each aspect of everyday life was full of stress - from propaganda pouring out of mass media to long lines forming in fronts of shops due to the general shortage of food and industrial goods. But Janusz did not yield. Whenever the weather looked promising he departed to Ostrowik, to collect data for his doctorate. His Ph.D. thesis Observational Aspects of the Evolution of Contact Binaries, written under the supervision of K. Stępień, was defended and published in 1985 [1].

The next two years Janusz spent together with his family in Baltimore, where he held a postdoc position at the Space Telescope Science Institute. He got acquainted with the then new observational technique - the CCD photometry - and quickly mastered it to perfection, becoming one of the best photometrists worldwide.

While at STScI, he proposed to observe eclipsing binary systems in globular clusters - a subject regarded widely as hopeless. His first applications for observing time were rejected. Instead of giving up he decided to focus on old open clusters first, and was granted appreciable amounts of time on the \#1 0.9-m telescope at Kitt Peak Observatory. The success came quickly - in late 1985 he discovered three new contact binaries in NGC 188 [2]. Since then the photometry of stellar clusters - both open and globular - became his main scientific specialty and his lifelong passion.

Back in Poland he was appointed as a researcher at the Warsaw University Observatory. In early 90s thanks to his photometric skills he became one of the founding fathers of the famous OGLE project which has brought innumerable discoveries in nearly all fields of astronomy. The launch of OGLE opened a long and very fruitful collaboration of Janusz with the Las Campanas Observatory which he was frequently visiting for the next two decades. It was at LCO where he discovered his first eclipsing binaries in a globular cluster - eight W UMa systems in NGC 4372 [3], and conceived the long-term project CASE (Cluster AgeS Experiment) conducted in collaboration with Carnegie Observatories.

Within CASE, he elaborated and implemented a method of age and distance determination for globular clusters based on observations of detached eclipsing binaries, originally proposed by Bohdan Paczynski. Together with his team he collected a unique set of data enabling to verify the theory of structure and evolution of Population II stars, and the determination of cluster membership from proper motions.

In 2000 he moved to the Nicolaus Copernicus Astronomical Center which granted him a full professorship. In the next year he became a member of the SSWG, serving the SALT 
community with his experience, engagement and - if necessary - criticism. Summarizing Janusz's activity in SSWG board, late Darragh O'Donoghue said: He was a firm supporter of SALT from the beginning, and an honest, even brutal, critic in latter years. I felt his criticism was a positive contribution.

He published nearly 300 papers and supervised eight Ph.D. students. His most important scientific achievement was the removal of a discrepancy between the cosmological age of the universe and the age of the oldest globular clusters [4]. In 2007 he obtained the prestigious award "Mistrz" (Master) from the Foundation for Polish Science.

\section{Scientific interests and achievements}

Janusz had multiple research interests, from photometry of various astronomical objects to spectroscopy of close binary systems as standard candles in globular cluster. His main research interests included i) evolutionary status of W UMa-type binaries; ii) CCD photometric observations of globular and old open clusters (GC and OC); iii) The Optical Gravitational Lensing Experiment (OGLE) and iv) The Cluster AgeS Experiment (CASE): age and distance of the globular clusters: $\omega$ Cen, 47 Tuc and old open cluster. Many of his projects were realized in collaboration with scientists from Canada, USA, Chile and various Polish institutions.

\subsection{Evolutionary status of W UMa-type binaries}

In his $\mathrm{PhD}$ thesis he derived absolute parameters of $18 \mathrm{~W}$ Uma-type binaries and discussed the evolutionary status of these systems based on mass-radius and mass-luminosity diagrams. He concluded that while the majority of stars from the analyzed sample lie close to the ZAMS in five on both diagrams, there is evidence that primary components of five systems deviate from relations fulfilled by typical main sequence stars.

\subsection{CCD photometry of old open and globular clusters}

Close binary stars or, more generally, binaries, drive the dynamical evolution of globular clusters. Their distribution within the cluster as well as processes leading to their destruction and/or ejection from the cluster are crucial to the understanding of secular changes in cluster's populations. During his stay at STScI Janusz started CCD observations of GCs and old open clusters in collaboration with M. Shara. Their obvious first target was NGC 188 because it hosted four known W UMa-type contact systems. In addition to the three W UMas mentioned above they found three stars to vary on a time scale of a few days; among them a likely FK Com variable. In a summary, the authors suggest that we may see, in one open cluster, the three important sequential stages in contact binary evolution: normal W UMa-type stars, an evolved longer-period binary and an FK Comae-type star.

His next attempt - a CCD search for faint variables in $\omega$ Cen and 47 Tuc - [5] was unsuccessful. No variables with B amplitudes greater than 0.003-0.008 mag were found among 2200 stars with $\mathrm{M} \leq+7.5$ in the field containing a low-luminosity X-ray source in the $\omega$ Cen nor among 1550 stars with similar luminosity in 47 Tuc. The space density of W UMa-type binaries in the outer parts of $\omega$ Cen and 47 Tuc was found to be at least as low as (and possibly up to eight times lower than) in the solar neighborhood. In another paper [6] Janusz searched six 
old and intermediate-age open clusters for contact binaries. Not a single W UMa binary was detected, while about three were expected among the 1600 stars examined. The authors concluded that contact binaries are at least as rare in the observed open clusters as they are in the field, and - again - that they may be significantly more rare. So far, NGC 188 remained unique in having seven W UMa stars among its 300 brightest members. Non-detection of even a single W UMa star among 545 members in the somewhat older cluster Mel 66 argued against great age being the parameter that determines whether a cluster is rich in contact binaries.

Photometry of other clusters (e. g., NGC 2112, NGC 2266, NGC 2355, NGC 2420, NGC 6791, NGC 7044, Be 32, King 2, K11, Be 39,Tombaugh 2) produced data enabling age and distance determination. Additionally, 18 short-period variables were found in Be 39 and Tombaugh 2; 12 of them being W UMa-type binaries. [7],[8].

\subsection{The Optical Gravitational Lensing Experiment - OGLE}

The possibility of using gravitational microlenses as a probe of the dark, unseen matter in our Galaxy was originally proposed by Paczynski [9],[10]. Because the probability of a star being microlensed at a given moment is extremely small, only a large scale photometric survey in dense stellar fields is suitable to search for microlensing events. Paczynski [9] proposed that if the Galactic halo is made of massive compact objects then any star in LMC or SMC has a chance of being strongly micro-lensed. The same phenomenon would also operate on stars in front of the Galactic Bulge.

In April 1992 a long-term observational project was sterted, called the Optical Gravitational Lensing Experiment (OGLE). Its ultimate aim was to detect a statistically significant number of microlensing events towards the Galactic Bulge. The 1992 observations represented a pilot microlensing survey of the Bulge, designed to test the ability to monitor millions of stars over a long period of time. The first OGLE paper [11] described the technical features of the project; in particular - an efficient method to reduce huge amounts of data in a near-real time. Less than a year later, the first candidate microlensing event in the direction of the Galactic Bulge was reported [12]. Presently OGLE detects such events at a rate of a few per day. The scientific yield of the project includes discoveries of extrasolar planets, Kuiper Belt objects, and innumerable variable stars of all kinds. It is far too large to be described here; interested readers are referred to the web site http://ogle.astrouw.edu.pl/.

\subsection{The DIRECT project}

From 1996 to 2006 Janusz was involved in DIRECT - another large-scale project whose aim was to determine direct distances to M31 and M33 - using detached eclipsing binaries and Cepheids [13].

These two galaxies are stepping stones to most of our current effort to under-stand the evolving universe at large scales. They are essential to the calibration of the extragalactic distance scale, and for constraining population synthesis models for early galaxy formation and evolution. There is one simple requirement for all this: accurate distances. Eclipsing binaries offer a single-step distance determination, and may therefore provide an accurate zero point calibration - a major step towards very accurate determination of the Hubble constant. Like in the case of OGLE, the search for suitable variables required extensive time-series photometry of 
large areas of the target galaxies, yielding magnitudes and positions for hundreds of thousands of stellar objects.

Several DIRECT papers co-authored by Janusz contained catalogs of variables in M33 and M31, and reported the first succesful distance determination to a detached eclipsing binary in M33 [14]. The binary consisted of two O7 main-sequence stars, with masses $33.4 \pm 3.5$ and $30.0 \pm 3.3 \mathrm{M}_{\odot}$, radii $12.3 \pm 0.4$ and 8.8 $\pm 0.3 \mathrm{R}_{\odot}$, and efefctive temperatures 37,000 \pm 1500 and $35,600 \pm 1500 \mathrm{~K}$. Using BVRJHKs photometry for the flux calibration, the authors obtained a distance modulus of $24.92 \pm 0.12 \mathrm{mag}(964 \pm 54 \mathrm{kpc})$. This pioneering result was based on observations obtained with 1.2-m FLWO, 2.1-m KPNO, 3.5-m WIYN, 8-m Gemini, and 10-m Keck II telescopes.

\subsection{The Cluster AgeS Experiment - CASE}

Parallel with DIRECT Janusz started his own long-term project CASE whose goal was to determine accurate ages and distances of globular clusters. In the 90s such an initiative was really timely, as reconciliation of globular cluster ages and the Hubble time was a primary issue of the observational astronomy. Heretofore, the largest uncertainty in the calculation of globular cluster ages from color-magnitude diagram data had been the distance error, followed by the stellar model uncertainties associated with the choice of the mixing length parameter. The hopes that Hipparcos would resolve the distance issue had not been fulfilled. For example, determinations of the distance modulus to 47 Tuc based on Hipparcos subdwarfs gave a value of 13.57 mag, while the value based on Hipparcos red clump giants was 13.32 mag. The most likely reason for the discrepancy was the comparison of nearby stars with distant stars, with imperfect understanding of the population effects.

CASE avoided these difficulties by measuring stellar masses and radii in DEBS - detached eclipsing binaries which at the same time are double-line spectroscopic systems. Their masses and radii can be measured directly with an accuracy on the order of 1-2\% for a wide range of spectral types. To attain such accuracy for a given system one needs: i) precise light curves with good coverage of both eclipses; ii) radial velocity curves allowing determination of K1 and K2 semi-amplitudes with an accuracy better than $1 \%$. From light curves solution one may determine: inclination $i$, relative radii $r_{1,2} / A$ and relative luminosities $l_{1,2}$. Once the inclination is known, from radial velocity curves one may obtain masses of the components and the orbital separation $A$ in absolute units. From the latter, absolute radii $R_{1,2}$ automatically follow.

Determination of bolometric luminosities is less direct. One possiblity is to estimate effective temperatures based on the dereddened color index or from the spectrum, and then use the Stefan-Boltzman law. Another way is to estimete distance and distance modulus using the infrared culor versus surface brightness relation, and then apply bolometric corrections to absolute visual magnitudes of the components $\mathrm{M}_{\mathrm{v} 1,2}$.

The first DEBS with the distance derived this way was OGLEGC 17 in $\omega$ Cen [15]. The bolometric luminosities of the components were derived using calibrations $T_{\text {eff }}=T_{\text {eff }}([\mathrm{Fe} / \mathrm{H}], B-$ $V)$ and $T_{\text {eff }}=T_{\text {eff }}([\mathrm{Fe} / \mathrm{H}], V-K)$. The age of this system, estimated from the (age - turnoff mass) relation and from the M-L diagram was found to be $9.1<t<16.7 \mathrm{Gyr}$ and $12.9<t<18.5 \mathrm{Gyr}$, respectively. Next year this result was refined by CASE [16] and, independently, by Chaboyer \& 
Krauss [17], yielding $11.8 \pm 0.6$ and $11.10 \pm 0.67 \mathrm{Gyr}$, respectively. The problem of the discrepancy between the Hubble time and the age of globular clusters was solved.

By now CASE has published numerous papers presenting catalogs of variable stars in various clusters, and several papers devoted to age and distance determination for $\omega$ Cen, 47 Tuc, M4, M55, NGC 6253 and NGC 6362. As a by-product, proper motion data for a few hundred thousand members of globular clusters were derived, and are being made available to the astronomical community. With a very few exceptions, masses and radii of the components of the surveyed DEBS are derived with an accuracy better than $1 \%$, allowing to estimate ages with an error margin of $\sim 1$ Gyr. For example, in M4 observations of V65, V66, and V69 are reported, with orbital periods of 2.29, 8.11, and 48.19 days, respectively. The masses of the components are $0.8035 \pm 0.0086$ and $0.6050 \pm 0.0044 \mathrm{M}_{\odot}$ for V65; $0.7842 \pm 0.0045$ and $0.7443 \pm 0.0042 \mathrm{M}_{\odot}$ for V66; and $0.7665 \pm 0.0053$ and $0.7278 \pm 0.0048 \mathrm{M}_{\odot}$ for V69. Their radii are $1.147 \pm 0.010$ and $0.6110 \pm 0.0092 \mathrm{R}_{\odot}$ for V66; $0.9347 \pm 0.0048$ and $0.8298 \pm 0.0053 \mathrm{R}_{\odot}$ for V66; and $0.8655 \pm 0.0097$ and $0.8074 \pm 0.0080 \mathrm{R}_{\odot}$ for V69. The distance to M4 was found to be $1.82 \pm 0.04 \mathrm{kpc}$ (in good agreement with recent estimates based on entirely different methods), and its most probable age to be $11.2-11.3$ Gyr [18].

In other clusters interesting patterns begin to emerge, which seem to suggest that we begin to directly observe the long-suspected non-coevality of star formation.

Unfortunately, Janusz did not live to see it...

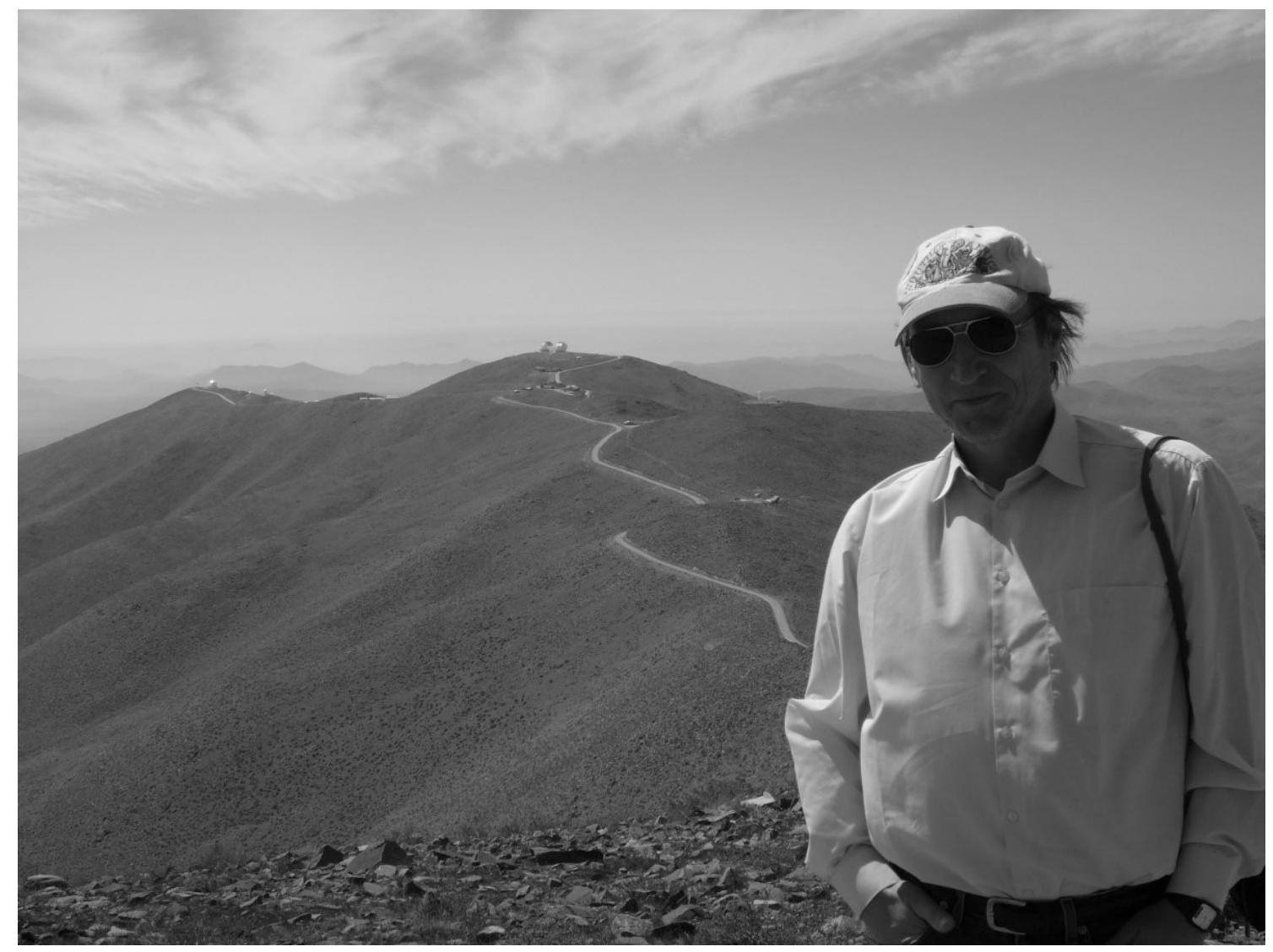




\section{In the eyes of friends}

At the telescope and at the computer he was extremely meticulous. Whatever was to be approved by him, had to be of highest quality. At the same time, he never stopped to be a true fan of astronomy for whom the starry sky is a source of strong emotions. Asked about his most pleasant memories from observing runs, Janusz said: I vividly remember the moment when I saw the zodiacal light for the first time. Most astronomy students know it from textbooks only, but at Las Campanas, where the sky is dark and very clear, one can see it with no difficulty. Even more splendidly looks the Sun when it sets straight into the Pacific, flashing that famous "green flash". And at the telescope? I always have a lot of satisfaction when an interesting binary whose properties I try to decipher behaves exactly according to my expectations. It happens at such moments that I jump out of joy in my chair.

Astronomy meant much for him, but he did not devote himself to it entirely. He was very fond of his three grandchildren, loved jazz and classical music, was a keen tourist and kayaker willingly spending time among friends. It is easy to imagine that he could prompt the Polish poet Wisława Szymborska (winner of the Nobel Prize) while she was writing

A new star has been discovered,

which doesn't mean that things have gotten brighter or that something we've been missing has appeared.

(...) The star's superb,

but that's no reason

why we can't drink to the ladies

who are incalculably closer. [19]

Janusz fell ill about 5 years ago and had to undergo treatment in various hospitals, but he was an impatient patient: he preferred to stay at home with his family and to work as long as it was only possible. At the beginning of 2015 his health rapidly deteriorated. For the last two months of his life he was too weak to come to the institute, but almost until his last day he continued working on M12 - the next CASE cluster.

Janusz passed away on March $6^{\text {th }}$, 2015. We lost an unequaled observer, and a warm and good-hearted friend full of humor.

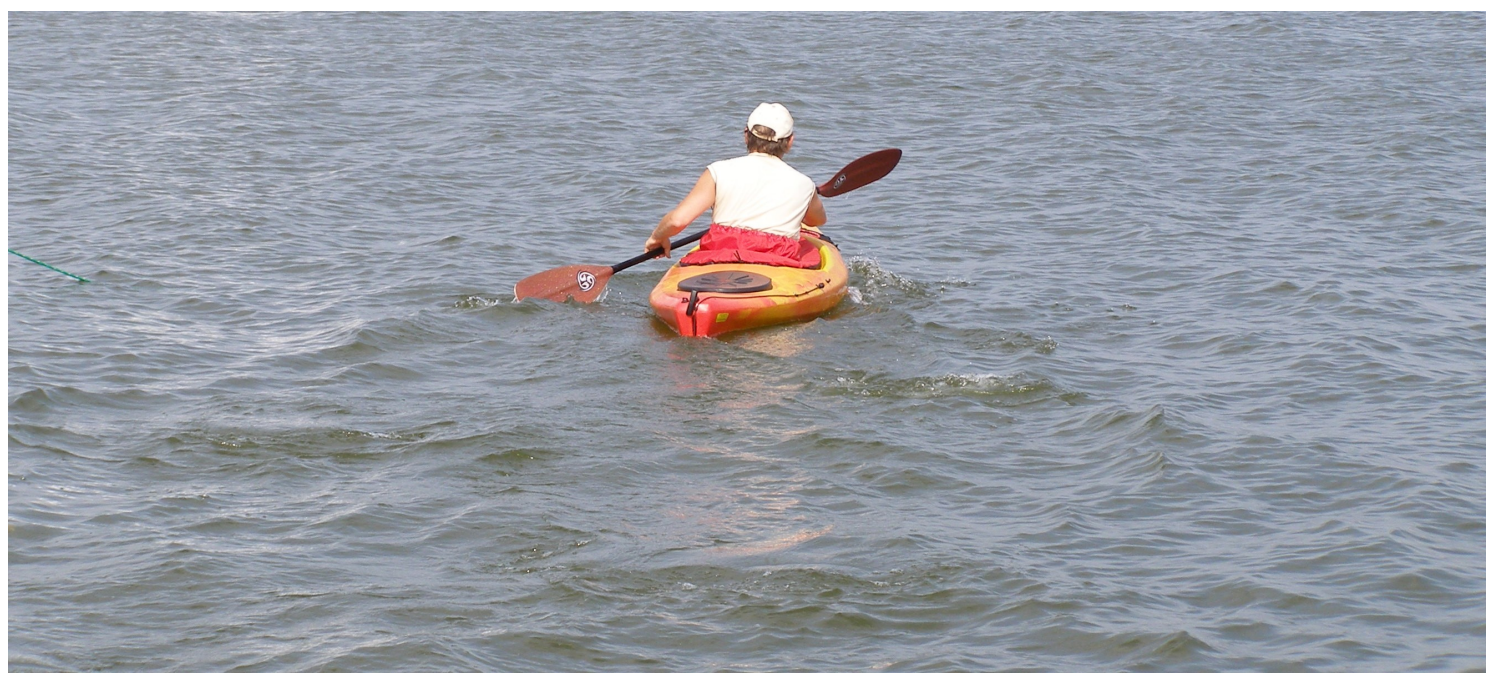




\section{References}

[1] J. Kaluzny, Evolutionary Status of W UMa-type Binaries, 1985, Acta Astron., 35, 313

[2] J. Kaluzny, M. Shara, The Discovery of Six New Short-period Variables in the Old Open Cluster NGC 188, 1987, ApJ, 314, 585

[3] J. Kaluzny, W. Krzeminski, Contact Binaries and SX Phe Variables in the Globular Cluster NGC 4372, 1993, MNRAS, 264, 785

[4] J. Kaluzny, I. Thompson, W. Krzemiński, et al., Photometry and Spectroscopy of Eclipsing Binaries in $\omega$ Centauri, 2002, in proceedings of $\omega$ Centauri: A Unique Window into Astrophysics, ASP Conference Series, 155

[5] M.Shara, J. Kaluzny, M. Potter, A. F. Moffat, A CCD search for faint variables in the field of and $\omega$ Centauri low-luminous X-ray source, and in 47 Tucanae, 1988, ApJ, 328, 594

[6] J. Kaluzny, M. Shara, A CCD survey for contact binaries in six open clusters, 1988, AJ, 95, 785

[7] J. Kaluzny, B. Mazur, W. Krzeminski, Discovery of 12 short-period eclipsing binaries in the old open cluster Berkeley 39, 1993, MNRAS, 262, 49

[8] M. Kubiak, J. Kaluzny, W. Krzeminski, M. Mateo, Mario Variable stars in the old open cluster Tombaugh 2 1992, AcA, 42, 155

[9] B. Paczynski, Gravitational Microlensing by the Galactic halo, 1986, ApJ 3041

[10] B. Paczynski, Gravitational Microlensing of the Galactic bulge stars, 1991, ApJ 371 L63

[11] A. Udalski, M. Szymanski, J. Kaluzny et al., The Optical Gravitational Lensing Experiment, 1992, Acta Atron., 42253

[12] A. Udalski, M. Szymanski, J. Kaluzny et al., The Optical Gravitational Lensing Experiment. Discovery of the First Candidate Micorlensing Event in the Direction of the Galactic Bulge, 1993, Acta Astron., 43289

[13] Macri, L. M.; Stanek, K. Z.; Sasselov, D. D.; Krockenberger, M.; Kaluzny, J. et al. The DIRECT Project: Catalogs of Stellar Objects in Nearby Galaxies. I. The Central Part of M33, 2001, AJ,121, 861

[14] A. Bonanos, K. Stanek, R. Kudritzki, L. Macri, D. Sasselov, J. Kaluzny, J. et al.,The First DIRECT Distance Determination to a Detached Eclipsing Binary in M33 2006, ApJ, 652, 313

[15] I. B. Thompson, J. Kałużny et al., Cluster AgeS Experiment (CASE): The age and distance of the globular cluster $\omega$ Centauri determined from observations of the eclipsing binary OGLEGC 17, 2001, AJ 1213089

[16] J. Kaluzny, I. B. Thompson, Photometry and Spectroscopy of Eclipsing Binaries in $\omega$ Centauri, 2002, in Omega Centauri, A Unique Window into Astrophysics, ASP Conference Proceedings, 265,.155

[17] B. Chaboyer, L. M. Krauss, Theoretical uncertainties in the subgiant mass-age relation and the absolute age of $\omega$ Centauri, 2002, ApJ, 567 L45

[18] J. Kaluzny et al., The Clusters Ages Experiment(CASE). V. Analysis of three eclipsing binaries in the globular cluster M4, 2013, AJ 14543

[19] W. Szymborska, Surplus, transl. S. Barańczak and C. Cavanagh, http://inwardboundpoetry.blogspot.com 